\title{
On Finite Integrals Involving Trigonometric, Bessel, and Legendre Functions
}

\author{
By Richard L. Lewis
}

Key Words: Finite Integrals, Bessel Functions, Legendre Functions, Generalized Hypergeometric Functions.

Abstract. A finite integral involving the product of powers of trigonometric functions, up to two associated Legendre functions, and zero or one Bessel function is evaluated. When certain combinations of the otherwise complex function parameters are integers, the resulting expression becomes greatly simplified. So restricting the parameters, this still quite general case may be transformed into four canonical forms, each of which admits rapid convergence of the only nonterminating series in the expressions. Finally, closed form expressions are obtained for a number of special cases.

In some recent work by the author on boundary-value problems, it became necessary to evaluate finite integrals containing the products of associated Legendre functions in the integrand. Due to the large amplitude oscillations of these functions over the interval of integration, numerical quadrature techniques proved unreliable.

A multiple series expression for the integral under consideration is presented, and then transforms of an otherwise rather untractable expression are introduced in order to obtain computable expressions when certain combinations of the parameters involved are integers. In general, however, the parameters $(\rho, \sigma, \nu, b, \beta, \eta, \lambda, \mu)$ have arbitrary complex values, provided they are so restricted as to allow the integral to exist. The integral considered then is

$$
I=\int_{0}^{1} x^{\rho}\left(1-x^{2}\right)^{\sigma} J_{\nu}\left(b\left(1-x^{2}\right)^{1 / 2}\right) P_{\beta}^{-\eta}(x) P_{\lambda}^{-\mu}(x) d x
$$

$$
\operatorname{Re}(\rho)>-1, \operatorname{Re}(\alpha)>-1,
$$

where $P_{\lambda}^{\mu}(x)$ is the associated Legendre function and $J_{\nu}(x)$ is the Bessel function of the first kind. The Bessel function is a limiting case of a Legendre function [1, Vol. II], hence its inclusion in the integrand. The necessary conditions for the existence of the integral are stated in (1), where the derived parameter is

$$
\alpha=\sigma+(\nu+\mu+\eta) / 2 .
$$

Upon substituting $x=\cos \theta$ in (1), the integrand becomes a product of powers of trigonometric functions, a Bessel function and two associated Legendre functions. Such a substitution will henceforth be considered obvious.

Since the formal derivation of the multiple series representation of (1) parallels

Received July 29, 1968. 
exactly the treatment by Barnes [2] of a simpler case, the details of the derivation are omitted. Briefly, the procedure is to express the integral (1) as a contour integral hugging the branch cut. The special functions are expressed as Mellin-Barnes type integrals [3]. The absolute convergence of such integrals has been proven by Dixon and Ferrar [4]; consequently, the orders of integration may be interchanged. Moving the outer contour integral inside the Mellin-Barnes integrals we obtain as the innermost integral Euler's first integral, which can be expressed as a product of gamma functions. Finally, the outer Mellin-Barnes integrals may be evaluated by summing their residues. Consequently, the starting point of our investigation is the representation of (1) as

$$
\begin{aligned}
I= & \frac{\Gamma\left(\frac{\rho+1}{2}\right) \Gamma(\alpha+1)\left(\frac{b}{2}\right)^{\nu}}{2^{\mu+\eta+1} \Gamma(\mu+1) \Gamma(\eta+1) \Gamma(\nu+1) \Gamma\left(\alpha+\frac{\rho+3}{2}\right)} \\
& \times \sum_{k=0}^{\infty} \frac{(\alpha+1)_{k}\left(-\frac{b^{2}}{4}\right)^{k}}{k !(\nu+1)_{k}\left(\alpha+\frac{\rho+3}{2}\right)_{k}} \\
& \times \sum_{j=0}^{\infty} \frac{\left(\frac{\eta-\beta}{2}\right)_{j}\left(\frac{\eta+\beta+1}{2}\right)_{j}(1+\alpha+k)_{j}}{j !(\eta+1)_{j}\left(\alpha+\frac{\rho+3}{2}+k\right)_{j}} \\
& \times{ }_{3} F_{2}\left\{\begin{array}{c}
\frac{\lambda+\mu+1}{2}, \frac{\mu-\lambda}{2}, 1+\alpha+k+j \\
\mu+1, \alpha+\frac{\rho+3}{2}+k+j
\end{array}\right\} .
\end{aligned}
$$

In the above, $\Gamma(z)$ is the well-known gamma function [1, Vol. I], and we have $(a)_{k}=a(a+1)(a+2) \cdots(a+k-1)$.

The generalized hypergeometric function is defined by the series expansion $[1$, Vol. I]

$$
{ }_{p} F_{q}\left(\begin{array}{c}
a_{1}, a_{2}, \cdots, a_{p} ; z \\
b_{1}, b_{2}, \cdots, b_{q}
\end{array}\right)=\sum_{k=0}^{\infty} \frac{\left(a_{1}\right)_{k}\left(a_{2}\right)_{k} \cdots\left(a_{p}\right)_{k}}{\left(b_{1}\right)_{k}\left(b_{2}\right)_{k} \cdots\left(b_{q}\right)_{k}} \frac{z^{k}}{k !} .
$$

In (2) we have adopted the standard convention that the argument $z$ of the hypergeometric function is omitted when it equals unity.

The representation (2) is a rather formidable result, but is interesting in that it is valid for arbitrarily complex values of the parameters, provided that the conditions for existence of the integral (1) are met. Numerically tractable cases can be obtained from (2) by placing further restrictions on the parameters involved. However, for full advantage of multiple parameter restrictions, use must be made of some unobvious multiple transformations which will allow (2) to be presented in a variety of highly utilizable forms. Indeed, a number of extremely unobvious closed form expressions will be presented as special cases.

The most obvious parameter restriction is to require that the difference $\lambda-\mu$ be 
an integer; however, $\lambda$ and $\mu$ themselves may still be complex. Such a restriction is quite common. Indeed, if $m, n$ are positive integers we have the associated Legendre polynomials,

$$
P_{n}{ }^{m}(x)=(-)^{m} \frac{(n+m) !}{(n-m) !} P_{n}{ }^{-m}(x),
$$

and the Gegenbauer polynomials [1, Vol. I],

$$
C_{n}{ }^{\nu}(x)=\frac{(2 \nu)_{n}}{n !} \Gamma\left(\nu+\frac{1}{2}\right)\left(\frac{1-x^{2}}{4}\right)^{1 / 4-D / 2} P_{\nu-1 / 2+n}^{1 / 2-\nu}(x) .
$$

The importance of (3.b) arises when considering integrals of the generating function

$$
\left(1-2 h x+h^{2}\right)^{-\nu}=\sum_{n=0}^{\infty} C_{n}^{\nu}(x) h^{n} .
$$

Other special functions that can be expressed as associated Legendre functions with an integer difference $\lambda-\mu$ include the Chebyshev polynomials and also a special case of the Jacobi polynomials [5].

We shall require two identities [6, Section 3.5]

$$
{ }_{3} F_{2}\left(\begin{array}{c}
a, b, c \\
e, f
\end{array}\right)=\frac{\Gamma(e+f-a-b-c) \Gamma(f)}{\Gamma(f-c) \Gamma(e+f-a-b)}{ }_{3} F_{2}\left(\begin{array}{c}
e-b, e-a, c \\
e, e+f-a-b
\end{array}\right)
$$

and [7, Eq. 23]

$$
\begin{aligned}
{ }_{3} F_{2}\left(\begin{array}{c}
a, b,-m \\
e, f
\end{array}\right)= & (-)^{m} \frac{\Gamma(1-e+b) \Gamma(f-b+m) \Gamma(e) \Gamma(f)}{\Gamma(1-e+b-m) \Gamma(f-b) \Gamma(e+m) \Gamma(f+m)} \\
& \times{ }_{3} F_{2}\left(\begin{array}{c}
1-s, b,-m \\
1-e+b-m, 1-f+b-m
\end{array}\right),
\end{aligned}
$$

where $m$ is an integer, $s=e+f-a-b+m$, and the other parameters are arbitrary. Note that when $s$ is equal to unity (5) reduces to Saalschütz's theorem [8] (cf. [6]). Using these identities, we express (2) in terms of four canonical forms. In the event that $(\lambda-\mu) / 2=n$, an integer, we have (6.a) and (6.b).

Note that in both expressions the first sum terminates at the $n$th term. The value in having two expressions to represent the same case arises when $\beta-\eta$ is also an integer, for then the second series will also terminate in one or the other of these two representations. Thus, if $(\beta-\eta) / 2=m$, the second series in (6.a) will terminate at the $m$ th term, while if $(\beta-\eta-1) / 2=m^{\prime}$, the second series in (6.b) will terminate at the $m^{\prime}$ th term.

Also of special importance is the case where $\rho$ is an integer. If $\rho$ is an even integer in (6.a) the first series will terminate at the smaller of $n, \rho / 2$; if $\rho$ is an odd integer in (6.b) the first series will terminate at the smaller of $n,(\rho+1) / 2$. We shall characterize this situation by saying that the series terminates early. If the second series terminates at $m$ in (6.a) or at $m^{\prime}$ in (6.b), then the two respective cases in which the first series terminates early also correspond to the integrand in (1) being an even function of the variable of integration.

We next have the case that $\lambda-\mu$ is odd. Thus, when $(\lambda-\mu-1) / 2$ is the integer $n$, we have (7.a) and (7.b). 

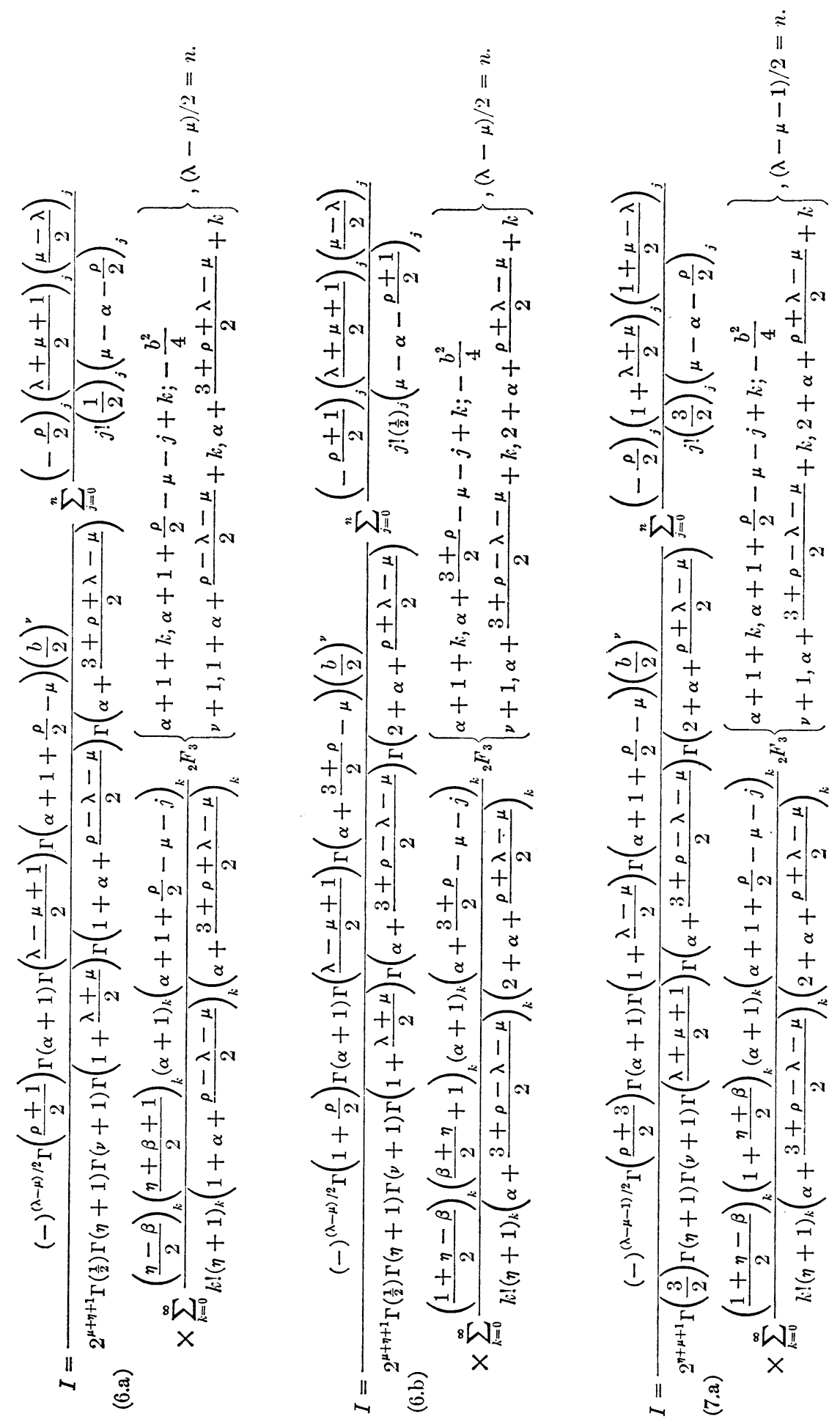
ON FINITE INTEGRALS

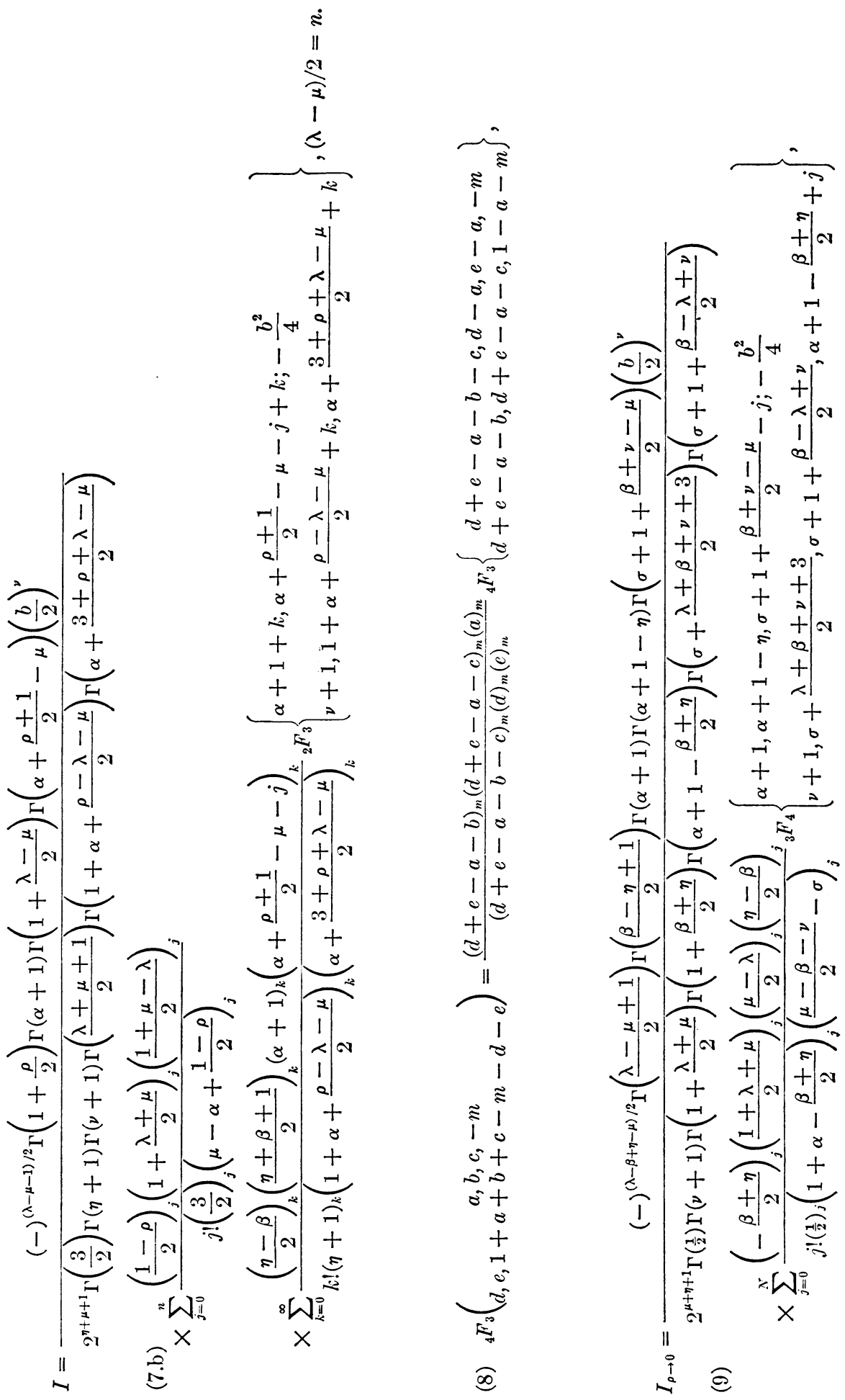


The same general comments that applied to Eqs. (6) apply here as well. Thus, the second series in (7.a) terminates at the $m^{\prime}$ th term whenever $m^{\prime}=(\beta-\eta-1) / 2$ is an integer, whereas in (7.b) the second series terminates at the $m$ th term whenever $m=(\beta-\eta) / 2$ is an integer. When the integrand (1) is an even function of its argument, then the second series in Eqs. (7) will not only terminate, but also the first series will terminate early. Early termination of the first series in (7.a)/(7.b) corresponds to $\rho$ assuming even/odd integer values, respectively.

The first special case to be considered is $\rho=0$. From [9], we have the identity (8). We obtain Eq. (9) by reversing the order of summation in (6.a), applying (8), and then changing back the order of summation. In $(9)$, both $(\lambda-\mu) / 2$ and $(\beta-\eta) / 2$ are restricted to integer values, and $N$ is the smaller of the two. Similarly, from (7.a) we obtain (10) for the case where both $\lambda-\mu$ and $\beta-\eta$ are odd integers. In (10), $M$ is the smaller of $(\lambda-\mu-1) / 2,(\beta-\eta-1) / 2$. The advantage of (9) and (10) over the original expressions (6.a) and (7.a) is that the 3-4 generalized hypergeometric series converge faster than the $2-3$ series. Indeed, one can apply an elementary error estimation analysis (such as in [10]) to the truncated hypergeometric series to show that after $m$ terms the error in the hypergeometric series on the right of (9) is proportional to

$$
E_{m} \propto\left(\frac{b \cdot e}{2 m}\right)^{2 m} \times\left(\frac{m}{e}\right)^{-(2 j+\nu+3 / 2)} .
$$

Thus, with each succeeding term of the terminating series, fewer terms of the corresponding hypergeometric series need to be summed. A similar analysis applied to (6.a) gives the result

$$
E_{m} \propto\left(\frac{b \cdot e}{2 m}\right)^{2 m}\left(\frac{m}{e}\right)^{-(\nu+3 / 2)} .
$$

In addition to the case $\rho=0$ we should like to treat the case $\rho=1$, for then we could compute all cases in which $\rho$ assumes positive integer values and the integrand in (1) is an even function of the variable of integration. That is, for $\rho \geqq 2$, we can use repeatedly the elementary relation $\cos ^{2} \theta=1-\sin ^{2} \theta$.

The identity (8) was adequate for obtaining an inner series which converges faster than the original inner series when we applied it to (6.a) and (7.a), but it cannot be used to transform (6.b) and (7.b) since there the difference in the sums of the denominator terms and the numerator terms in the second series is not unity.

We can obtain a modification of the result (8), however, by equating the coefficients of $\zeta^{n}$ in (11), cf. [6], where $s=u+v+w-x-y-z+n$. Thus, when $s=1$, 2 we obtain (12). When $s=1$ in (12), we can obtain (8) by applying the identity to itself. Using (12), (6.b) becomes (13), where $L$ is the smaller of the two positive integers, $(\lambda-\mu) / 2$ and $(\beta-\eta-1) / 2$. From (7.b) we obtain (14), where $K$ is the smaller of the two positive integers, $(\lambda-\mu-1) / 2$ and $(\beta-\eta) / 2$. In each of the results (9), (10), (13), and (14) the truncation error after $m$ terms of the infinite series is smaller than the truncation error after $m$ terms in the original representations by a factor $m^{-2 j}$, where $j$ designates the term of the terminating series. 

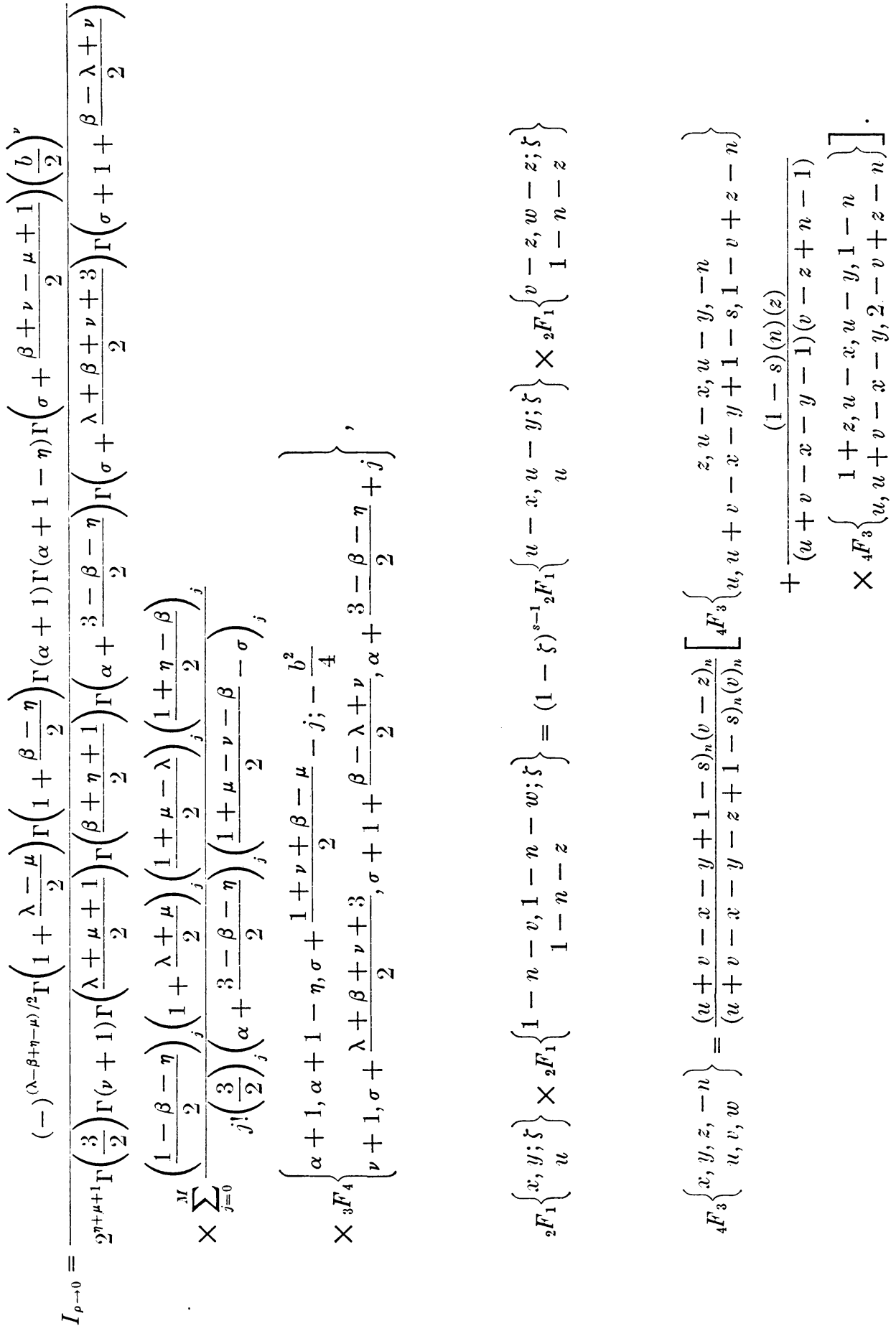

ఏ

ミ 


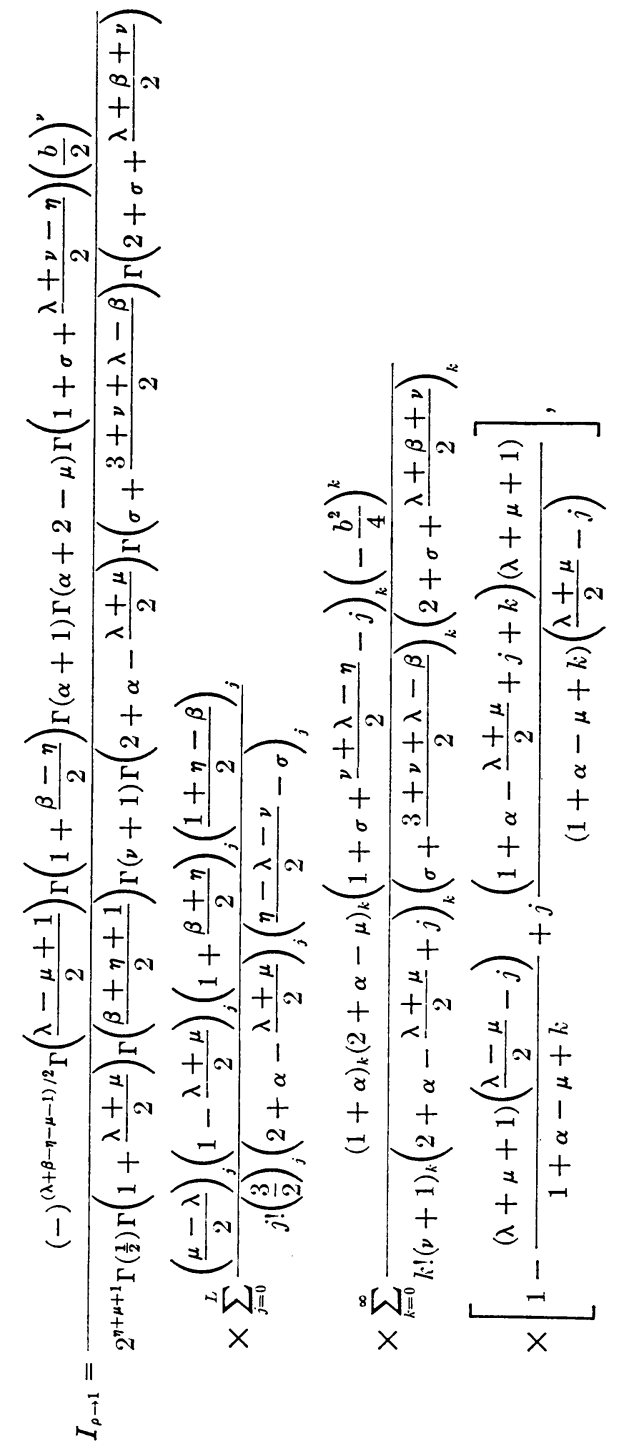

$\widehat{\varrho a}$

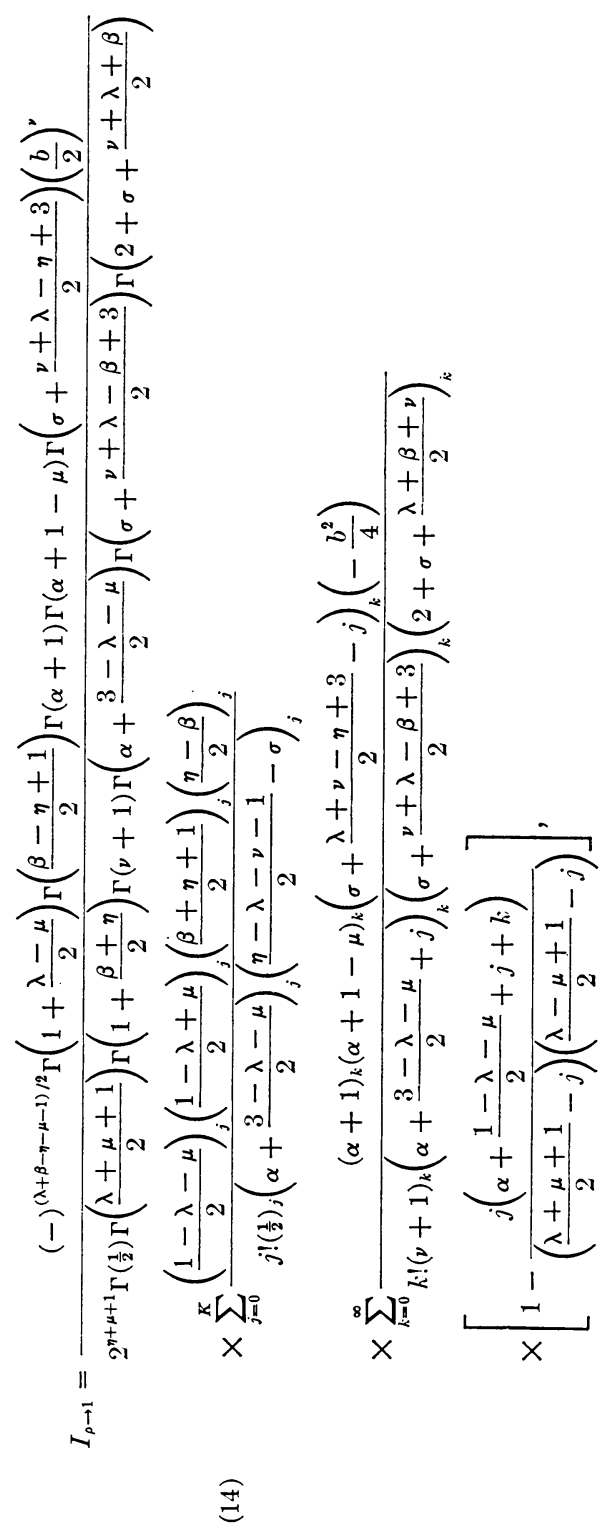



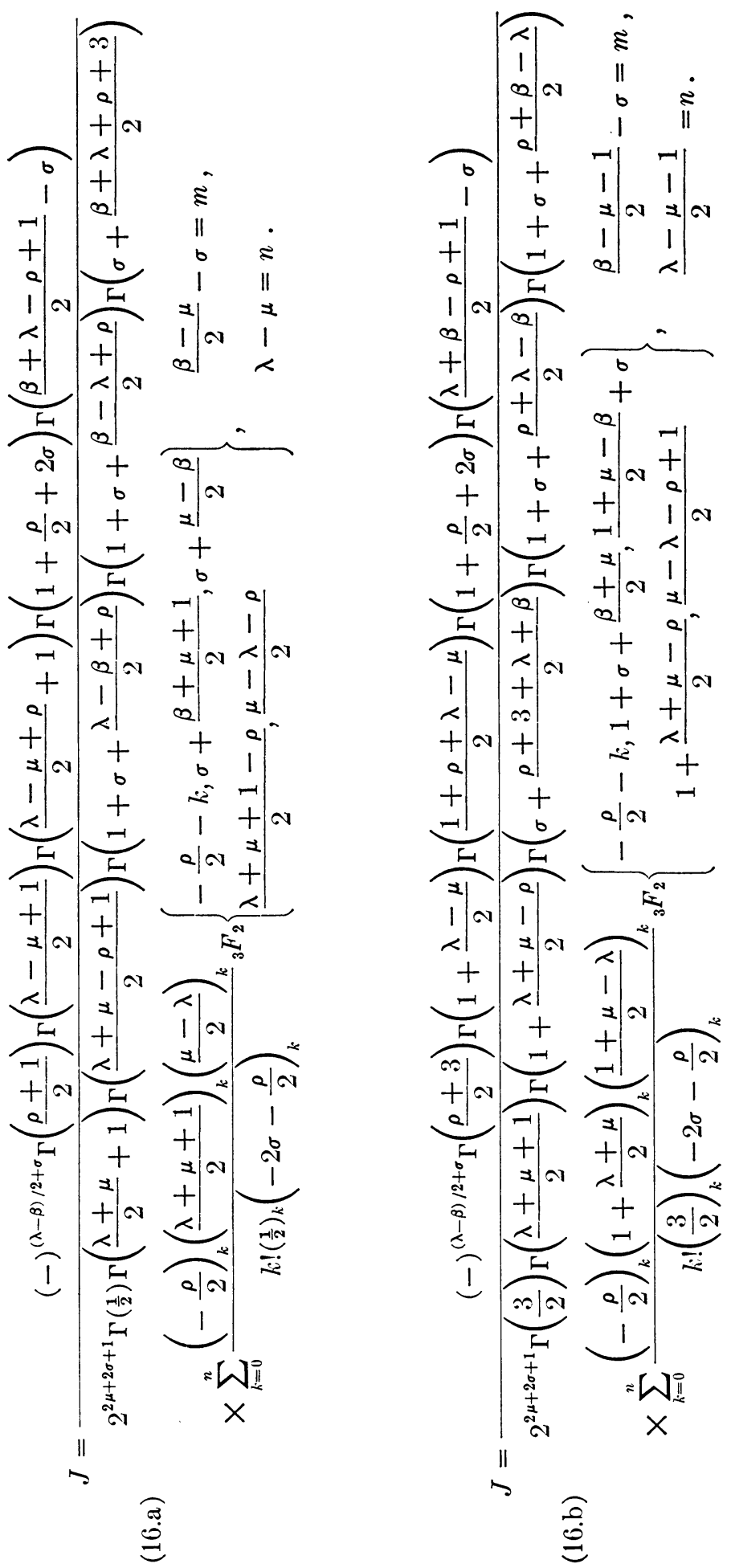
We next turn to the situation where one of the special functions in (1) disappears. If we set $\beta=\eta$ in (6.a) and $\beta=\eta+1$ in (6.b), so that the first associated Legendre function in (1) becomes the product of $\left(1-x^{2}\right)^{\eta / 2}$ times unity and $x$, respectively, then Eqs. (6) will coalesce into identical results. A similar statement is true for Eqs. (7). Finally if the second associated Legendre function is allowed to disappear, Eqs. (6) and (7) coalesce into the following well-known Riemann-Liouville integral [11, Eq. 65, Chapter 13].

$$
\begin{gathered}
\int_{0}^{1} y^{\sigma}(1-y)^{(\rho-1) / 2} J_{\nu}(b \sqrt{ } y) d y=\frac{\Gamma\left(\frac{\rho+1}{2}\right) \Gamma\left(\sigma+\frac{\nu}{2}+1\right)\left(\frac{b}{2}\right)^{\nu}}{\Gamma(\nu+1) \Gamma\left(\sigma+\frac{\rho+\nu+3}{2}\right)} \\
\cdot{ }_{1} F_{2}\left\{\begin{array}{l}
\sigma+\frac{\nu}{2}+1 ;-\frac{b^{2}}{4} \\
\left.\nu+1, \sigma+\frac{\rho+\nu+3}{2}\right\}
\end{array}\right.
\end{gathered}
$$

More interesting situations occur when $\nu=b=0$. Let us define

$$
J \equiv \int_{0}^{1} x^{\rho}\left(1-x^{2}\right)^{\sigma} P_{\beta}^{-\mu-2 \sigma}(x) P_{\lambda}^{-\mu}(x) d x, \quad \operatorname{Re}(\rho)>-1, \operatorname{Re}(\mu+2 \sigma)>-1,
$$

Then, if we set $\alpha=\eta=\mu+2 \sigma$ and require that $\beta-\eta$ be an integer, we can again use (5) and obtain (16.a) from (6.a) and (16.b) from (7.a).

Here $m, n$ are restricted to nonnegative integer values. This method of denoting such quantities is used throughout.

Note that the hypergeometric series in (16.a) and (16.b) terminate after $m$ terms, and that the first sum terminates after $n$ terms. If $\rho$ is an even integer, both the hypergeometric functions and the series terminate early. In particular, for $\rho=0$, the two equations coalesce into

$$
\begin{aligned}
& \int_{-1}^{1}\left(1-x^{2}\right)^{\sigma} P_{\beta}^{-\mu-2 \sigma}(x) P_{\lambda}^{-\mu}(x) d x \\
= & \frac{(-)^{(\lambda-\beta) / 2+\sigma} \Gamma(\lambda-\mu+1) \Gamma(2 \sigma+1) \Gamma\left(\frac{\lambda+\beta+1}{2}-\sigma\right)}{2^{2 \sigma} \Gamma(\lambda+\mu+1) \Gamma\left(\sigma+\frac{\lambda+\beta+3}{2}\right) \Gamma\left(1+\sigma+\frac{\lambda-\beta}{2}\right) \Gamma\left(1+\sigma+\frac{\beta-\lambda}{2}\right)} .
\end{aligned}
$$

The only restrictions on (17) are that $\operatorname{Re}(\mu+2 \sigma)>-1$, and that the differences $\lambda-\mu$ and $\beta-\mu-2 \sigma$ both be integers. Note that the extension of the interval of integration implies that the integrand be an even function of $x$ in order to yield a nontrivial result. Equation (17) is a generalization of a result that restricted $\sigma / 2$ to integer values [12].

Two special cases of (17) require immediate mention. If $\lambda-\mu$ is an even integer, set $\beta=\mu+2 \sigma$ to obtain 

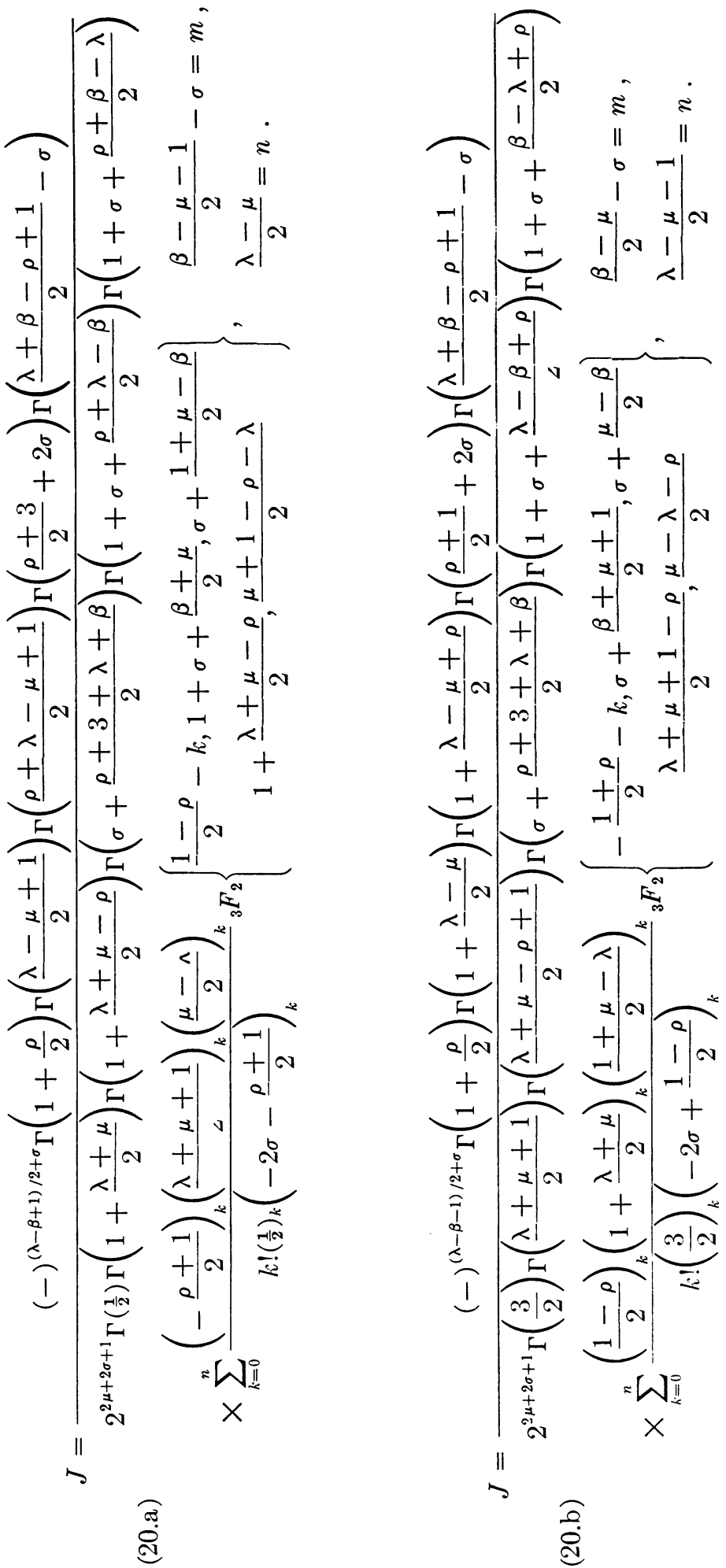


$$
\begin{gathered}
\int_{0}^{1}\left(1-x^{2}\right)^{\sigma} P_{\lambda}^{-\mu}(x) d x=\frac{\Gamma\left(\frac{\lambda-\mu+1}{2}\right) \Gamma\left(1+\sigma+\frac{\mu}{2}\right) \Gamma\left(\frac{\lambda}{2}-\sigma\right)}{2^{\mu+1} \Gamma\left(1+\frac{\lambda+\mu}{2}\right) \Gamma\left(\frac{\lambda+3}{2}+\sigma\right) \Gamma\left(\frac{\mu}{2}-\sigma\right)} \\
\frac{\lambda-\mu}{2}=n
\end{gathered}
$$

whereas if $\lambda-\mu$ is an odd integer, set $\beta=\mu+2 \sigma+1$ to obtain

$$
\begin{array}{r}
\int_{0}^{1} x\left(1-x^{2}\right)^{\sigma} P_{\lambda}^{-\mu}(x) d x=\frac{\Gamma\left(1+\frac{\lambda-\mu}{2}\right) \Gamma\left(\sigma+\frac{\mu}{2}+1\right) \Gamma\left(\frac{\lambda-1}{2}-\sigma\right)}{2^{\mu+1} \Gamma\left(\frac{\lambda+\mu+1}{2}\right) \Gamma\left(2+\sigma+\frac{\lambda}{2}\right) \Gamma\left(\frac{\mu}{2}-\sigma\right)} \\
\frac{\lambda-\mu-1}{2}=n
\end{array}
$$

The integrability conditions for the results in (18) and (19) reduce to $\operatorname{Re}(\sigma+\mu / 2)$ $>-1$.

Now upon applying (5) to (6.b) and (7.b) we obtain (20.a) and (20.b), respectively. As before, the first sum terminates after $n$ terms, the hypergeometric function terminates after $m$ terms, and all the series involved will terminate early if $\rho$ is an odd integer. As a check on the validity of Eqs. (20), note that if $\beta$ is set equal to $2 \sigma+\mu+1$ in Eq. (20.a), and if $\beta$ is set equal to $2 \sigma+\mu$ in Eq. (16.a), then these two equations will coalesce into identical results. A similar statement can be made for Eqs. (16.b) and (20.b). In particular Eq. (19) follows immediately from (20.b) when one sets $\beta=\mu+2 \sigma$ and $\rho=1$.

Comments regarding other special cases that arise from Eqs. (6) and (7) are similar to those already given. Consequently, only the results will be given, along with the necessary restrictions. It is important to emphasize, however, that $m, n$ represent nonnegative integers in the following.

Each of the following ten special cases can be obtained from Eqs. (6) and (7) by selecting the parameters in the second series so that one numerator and one denominator term cancel, and then summing the resultant 3-2 hypergeometric series by means of the identity (5). In each instance $\eta-\beta$ is a negative integer.

$$
\int_{-1}^{1}\left(1-x^{2}\right)^{\sigma} P_{\beta}^{-2 \sigma+\mu}(x) P_{\lambda}^{-\mu}(x) d x=\frac{(-)^{(\lambda-\beta) / 2+\sigma-\mu} \Gamma(1+2 \sigma) \Gamma\left(\frac{\lambda+\beta+1}{2}-\sigma\right)}{2^{2 \sigma} \Gamma\left(1+\sigma+\frac{\lambda-\beta}{2}\right) \Gamma\left(1+\sigma+\frac{\beta-\lambda}{2}\right) \Gamma\left(\sigma+\frac{\beta+\lambda+3}{2}\right)},
$$

where $\lambda-\mu=n, \beta+\mu-2 \sigma=m, \operatorname{Re}(\sigma)>-\frac{1}{2}$;

$$
\begin{aligned}
& \int_{0}^{1} x\left(1-x^{2}\right)^{\sigma} P_{\beta}^{-2 \sigma+\mu}(x) I_{\lambda}{ }^{-\mu}(x) d x \\
& =\frac{(-)^{(\lambda+\beta-1) / 2-\sigma} \Gamma(1+2 \sigma) \Gamma\left(\frac{\lambda+\beta}{2}-\sigma\right)\left[\left(\frac{\lambda-\mu}{2}\right)\left(\frac{\lambda+\mu+1}{2}\right)-\left(\frac{\beta+\mu}{2}-\sigma\right)\left(\frac{\beta-\mu+1}{2}+\sigma\right)\right]}{2^{2 \sigma+1} \Gamma\left(\sigma+\frac{3+\lambda-\beta}{2}\right) \Gamma\left(\sigma+\frac{3+\beta-\lambda}{2}\right) \Gamma\left(2+\sigma+\frac{\lambda+\beta}{2}\right)},
\end{aligned}
$$


where $(\lambda-\mu-1) / 2=n,(\beta+\mu) / 2-\sigma=m, \operatorname{Re}(\sigma)>-\frac{1}{2}$;

$$
\int_{-1}^{1}\left(1-x^{2}\right)^{\sigma} \Gamma_{\beta}^{-\mu-2 \sigma-2}(x) P_{\lambda}^{-\mu}(x) d x=2^{2 \sigma+2} \frac{\Gamma(\beta-\mu-2 \sigma-1) \Gamma(2+2 \sigma+\mu)}{\Gamma(\mu+1) \Gamma(\beta+\mu+2 \sigma+3)},
$$

where $\lambda-\mu=n, \beta-\mu-2 \sigma-2=m$, $\operatorname{Re}(1+\mu+2 \sigma)>-1$, and furthermore $\beta \geqq \lambda+2 \sigma+2$;

$$
\int_{-1}^{1}\left(1-x^{2}\right)^{\sigma} P_{\lambda-2 \sigma}^{-\eta}(x) P_{\lambda}^{\lambda^{-\mu}}(. x) d x=\frac{(-)^{(\eta-\mu) / 2+\sigma} \Gamma(\lambda-\mu+1) \Gamma\left(\lambda+\frac{1}{2}-2 \sigma\right)}{2^{2 \sigma} \Gamma(\lambda+\eta+1-2 \sigma) \Gamma\left(\lambda+\frac{3}{2}\right)}
$$

where $\lambda-\mu=n, \lambda-\eta-2 \sigma=m, \operatorname{Re}(\sigma+(\eta+\mu) / 2)>-1$;

$$
\begin{aligned}
& \int_{-1}^{1}\left(1-x^{2}\right)^{\sigma} P_{\lambda+2 \sigma+2}^{-\eta}(x) P_{\lambda}^{-\mu}(x) d x \\
& =(-)^{(\eta-\mu) / 2-\sigma-1} 2^{2 \sigma+2} \frac{\Gamma(\lambda-\mu+1) \Gamma\left(\sigma+1+\frac{\eta+\mu}{2}\right) \Gamma\left(\sigma+1+\frac{\eta-\mu}{2}\right)}{\Gamma(\lambda+2 \sigma+\eta+3) \Gamma\left(\frac{\eta+\mu}{2}-\sigma\right) \Gamma\left(\frac{\eta-\mu}{2}-\sigma\right) \Gamma(2+2 \sigma)},
\end{aligned}
$$

where $\lambda-\mu=n, \lambda-\eta+2 \sigma+2=m, \operatorname{Re}(\sigma+(\eta+\mu) / 2)>-1$;

$$
\begin{aligned}
\int_{0}^{1} x\left(1-x^{2}\right)^{\sigma} P_{\lambda+2 \sigma+3}^{-\eta}(x) P_{\lambda}{ }^{-\mu}(x) d x & \\
= & (-)^{(\eta-\mu) / 2-\sigma \cdot 2^{2 \sigma+2}} \frac{\Gamma(\lambda-\mu+1) \Gamma\left(\sigma+1+\frac{\eta+\mu}{2}\right) \Gamma\left(\sigma+1+\frac{\eta-\mu}{2}\right)}{\Gamma(\lambda+\eta+2 \sigma+4) \Gamma\left(\frac{\eta+\mu}{2}-\sigma\right) \Gamma\left(\frac{\eta-\mu}{2}-\sigma\right) \Gamma(3+2 \sigma)} \\
& \times\left[\left(\frac{\lambda-\mu+1}{2}\right)\left(\frac{\eta+\mu}{2}-\sigma-1\right)-\left(\frac{\lambda-\eta+3}{2}+\sigma\right)\left(\sigma+1+\frac{\eta+\mu}{2}\right)\right],
\end{aligned}
$$

where $(\lambda-\mu-1) / 2=n,(\lambda-\eta+3) / 2+\sigma=m, \operatorname{Re}(\sigma+(\eta+\mu) / 2)>-1$;

$$
\begin{aligned}
& \int_{0}^{1} x\left(1-x^{2}\right)^{\sigma} P_{\lambda+2 \sigma+3}^{-\eta}(x) P_{\lambda}^{-\mu}(x) d . v \\
& =(-)^{(\eta-\mu) / 2-\sigma-1} 2^{2 \sigma+2} \frac{\Gamma(\lambda-\mu+1) \Gamma\left(\sigma+1+\frac{\eta+\mu}{2}\right) \Gamma\left(\sigma+1+\frac{\eta-\mu}{2}\right)}{\Gamma(\lambda+2 \sigma+\eta+4) \Gamma\left(\frac{\eta+\mu}{2}-\sigma\right) \Gamma\left(\frac{\eta-\mu}{2}-\sigma\right) \Gamma(2 \sigma+3)} \\
& \quad \times\left[\frac{\left(\frac{\eta+\mu}{2}-\sigma-1\right)\left(\frac{\eta-\mu}{2}+\sigma+1\right)}{\lambda+\mu}-\left(\frac{\eta+\mu}{2}-\sigma-1\right)\left(\frac{\lambda-\mu}{2}\right)\left(\frac{\lambda+\mu+1}{\lambda+\mu}\right)\right. \\
& \left.+\left(\sigma+1+\frac{\eta+\mu}{2}\right)\left(\sigma+1+\frac{\lambda-\eta}{2}\right)\left(\frac{\lambda+\mu+1}{\lambda+\mu}\right)\right],
\end{aligned}
$$


where $(\lambda-\mu) / 2=n,(\lambda-\eta) / 2+\sigma+1=m, \operatorname{Re}(\sigma+(\eta+\mu) / 2)>-1$;

$$
\begin{aligned}
& \int_{-1}^{1}\left(1-x^{2}\right)^{\sigma} P_{2 \sigma+1-\lambda}^{-\eta}(x) P_{\lambda}^{-\mu}(x) d x \\
& =(-)^{\lambda-\sigma+(\eta-\mu-1) / 2} \frac{2^{2 \sigma+1} \Gamma\left(1+\sigma+\frac{\eta+\mu}{2}\right) \Gamma\left(1+\sigma+\frac{\eta-\mu}{2}\right) \Gamma\left(1+\sigma+\frac{\mu-\eta}{2}\right) \Gamma\left(1+\sigma-\frac{\eta+\mu}{2}\right)}{\pi \Gamma(\lambda+\mu+1) \Gamma(2 \sigma+2+\eta-\lambda) \Gamma(2 \sigma+2)},
\end{aligned}
$$

where $\lambda-\mu=n, 2 \sigma+1-\lambda-\eta=m, \operatorname{Re}(\sigma+(\eta+\mu) / 2)>-1$;

$$
\begin{aligned}
\int_{0}^{1} x(1 & \left.-x^{2}\right)^{\sigma} P_{2 \sigma+2-\lambda}^{-\eta}(x) P_{\lambda}^{\lambda^{-\mu}(x) d x} \\
= & (-)^{(\eta+\mu-1) / 2-\sigma} \frac{2^{2 \sigma+1} \Gamma\left(1+\sigma+\frac{\eta+\mu}{2}\right) \Gamma\left(1+\sigma+\frac{\eta-\mu}{2}\right) \Gamma\left(1+\sigma+\frac{\mu-\eta}{2}\right) \Gamma\left(1+\sigma-\frac{\eta+\mu}{2}\right)}{\pi \Gamma(\lambda+\mu+1) \Gamma(2 \sigma+3+\eta-\lambda) \Gamma(3+2 \sigma)} \\
& \times\left[\left(1+\sigma-\frac{\eta+\mu}{2}\right)\left(\frac{\lambda+\mu}{2}\right)-\left(1+\sigma-\frac{\lambda+\eta}{2}\right)\left(1+\sigma+\frac{\eta+\mu}{2}\right)\right],
\end{aligned}
$$

where $(\lambda-\mu-1) / 2=n, 1+\sigma-(\lambda+\eta) / 2=m, \operatorname{Re}(\sigma+(\eta+\mu) / 2)>-1$;

$$
\begin{aligned}
& \int_{0}^{1} x\left(1-x^{2}\right)^{\sigma} P_{2 \sigma+2-\lambda}^{-\eta}(x) P_{\lambda}^{-\mu}(x) d x \\
& \left.=(-)^{(\eta+\mu-1) / 2-\sigma} \frac{2^{2 \sigma+1} \Gamma\left(1+\sigma+\frac{\eta+\mu}{2}\right) \Gamma\left(1+\sigma+\frac{\eta-\mu}{2}\right) \Gamma\left(1+\sigma+\frac{\mu-\eta}{2}\right) \Gamma\left(1+\sigma-\frac{\eta+\mu}{2}\right)}{\pi \Gamma(\lambda+\mu+1) \Gamma(2 \sigma+3+\eta-\lambda) \Gamma(3+2 \sigma)}\right) \\
& \times\left[\frac{\left(1+\sigma-\frac{\eta+\mu}{2}\right)\left(1+\sigma+\frac{\eta-\mu}{2}\right)}{\lambda-\mu+1}-\left(1+\sigma-\frac{\eta+\mu}{2}\right)\left(\frac{\lambda+\mu+1}{2}\right)\left(\frac{\lambda-\mu}{\lambda-\mu+1}\right)\right. \\
& \left.+\left(1+\sigma+\frac{\eta+\mu}{2}\right)\left(\sigma+\frac{1-\lambda-\eta}{2}\right)\left(\frac{\lambda-\mu}{\lambda-\mu}\right)\right],
\end{aligned}
$$

where $(\lambda-\mu) / 2=n, \sigma+(1-\lambda-\eta) / 2=m, \operatorname{Re}(\sigma+(\eta+\mu) / 2)>-1$.

The author has attempted to list all nontrivial special cases resulting from Eqs. (6) and (7) by equating numerator and denominator terms in the second series. Also considered were the additional special cases that resulted when the identity (8) was used to modify these numerator and denominator terms. It is possible that still more special cases can be obtained by using the transformations discovered by Sears [13] to modify the numerator and denominator terms in the second series. However, since each of Sears' transformations result in a 4-3 generalized hypergeometric series being transformed into a sum of three other hypergeometric series of the same type, calculations will be quite complex.

Acknowledgment. This work was sponsored by the Advanced Research Projects Agency and performed at the Environmental Science Services Administration, Institute for Telecommunication Sciences, Boulder, Colorado.

Ionospheric Telecommunications Laboratory

Environmental Science Services Administration

Boulder, Colorado 80302 
1. A. ErdéLYI, et al., Higher Transcendental Functions, Vols. I, II, McGraw-Hill, New York, 1953. MR 15, 419.

2. B. W. BARnes, "On generalized Legendre functions," Quart. J. Math. Oxford Ser., v. 31, 1908, pp. $97-204$.

3. B. I. Whittaker \& G. N. Watson, A Course of Modern Analysis, Cambridge Univ. Press, New York, 1927.

4. A. L. Dixon \& W. L. FerRar, Quart. J. Math. Oxford Ser., v. 7, 1936, pp. 81-96.

5. Milton Abramowitz \& I. A. Stegun, (Editors), Handbook of Mathematical Functions with Formulas, Graphs, and Mathematical Tables, Nat. Bur. Standards Appl. Math. Ser., 5.5, Superintendent of Documents, U. S. Government Printing Office, Washington, D. C., 1964; 3rd printing with corrections, 1965. MR 29 \#4914; MR 31 \#1400. 1935.

6. W. N. BaIley, Generalized Hypergeometric Series, Cambridge Univ. Press, New York,

7. F. J. W. Whipple, "A group of generalized hypergeometric series," Proc. London Math. Soc. (2), v. 23, 1925, pp. 104-114.

8. L. SaAlschüTZ, Zeitschrift für Math. und Phys., v. 35, 1890, pp. 186-188.

9. T. M. MACRoBert, "Formulae for generalized hypergeometric functions as particular cases of more general formulae," Philos. Mag., (7), v. 28, 1939, pp. 488-492.

10. M. MANGAD, "Some limiting values and two error estimation procedures for power series approximations," Math. Comp., v. 21, 1967, pp. 423-430.

11. A. ERdÉLYI, et al., Tables of Integral Transforms, Vol. II, McGraw-Hill, New York, 1954. MR 16, 468.

12. A. Gaunt, "The triplets of helium," Philos. Trans. Roy. Soc. Ser. A, v. 228, 1929, pp. $192-196$.

13. D. B. Sears, "The transformation theory of hypergeometric functions and cognate trigonometrical series," Proc. London Math. Soc., v. 53, 1951, pp. 138-157. MR 13, 33. 